Schmerz 2020 -34:197-199

https://doi.org/10.1007/s00482-020-00475-9

Online publiziert: 24. April 2020

(c) Springer Medizin Verlag $\mathrm{GmbH}$, ein Teil von Springer Nature 2020

Lukas Radbruch ${ }^{1,2} \cdot$ Hans-Georg Schaible ${ }^{3}$

${ }^{1}$ Klinik für Palliativmedizin, Universitätsklinikum Bonn, Bonn, Deutschland

${ }^{2}$ Zentrum für Palliativmedizin, Malteserkrankenhaus Bonn/Rhein-Sieg, Bonn/Rhein-Sieg, Deutschland

${ }^{3}$ Institut für Physiologie, Universität Jena, Jena, Deutschland

\title{
COVID-19 und Schmerz - Call for Papers
}

Während wir dies kurz vor Ostern schreiben, wird das Gesundheitswesen in Deutschland ebenso wie das gesamte öffentliche Leben von der CoronaPandemie und den Gegenmaßnahmen gegen die zu rasche Ausbreitung dominiert. Auch wenn Sie dies in einigen Tagen oder Wochen lesen, wird sich das noch nicht geändert haben, und es wird sicher noch länger dauern, bis wir zur Normalität zurückkehren können.

Die Pandemie nahm im Dezember 2019 ihren Anfang in China, Ende Januar 2020 wurden die ersten Erkrankungsfälle in Deutschland gemeldet und am 11. März 2020 erklärte die Weltgesundheitsorganisation die Corona-Epidemie zur Pandemie.

Trotz dieser sehr kurzen Zeitabfolgen sind bereits Publikationen zur Behandlung von Patienten mit COVID-19 erschienen. Neben den Empfehlungen zur intensivmedizinischen Behandlung [5] liegen auch erste Erfahrungsberichte zur Behandlung von belastenden Symptomen vor. In den Empfehlungen einer internationalen Arbeitsgruppe [1] wird die Häufigkeit von Luftnot mit 5-65\% der Patienten angegeben, während Husten bei $70-80 \%$ der Patienten berichtet wird. Angst und Delirium werden als weitere häufige Symptome aufgeführt, ohne dass dafür Prävalenzen genannt werden. In der symptomatischen Behandlung der Luftnot wird der Stellenwert von Opioiden betont, die bei Patienten mit COVID-19 effektiv die Luftnot verringern können. Schmerzen werden nicht als Symptom genannt.

Auch in der ersten deutschen Studie zur Behandlung von 50 Patienten mit COVID-19 aus Aachen [4] werden vor allem Fieber ( $82 \%$ der Patienten), Luftnot (48\%), Husten (42\%) und gastrointestinale Beschwerden (18\%, vor allem Diarrhö) berichtet. Schmerzen wurden nur von einzelnen Patienten als Muskelschmerzen (12\%), Halsschmerzen (4\%), Angina pectoris (4\%) und Kopfschmerzen $(2 \%)$ angegeben.

Bei Patienten mit schwerem Verlauf einer COVID-19-Infektion ist eine invasive Beatmung erforderlich, die in aller Regel mit einer ausreichenden Analgosedierung einhergeht. Bei zwei Patientengruppen darf die Notwendigkeit einer Symptomkontrolle aber nicht vernachlässigt werden. Bei Patienten mit schweren Begleiterkrankungen kann aufgrund der schlechten Erfolgsaussichten die Indikation einer intensivmedizinischen $\mathrm{Be}$ handlung und invasiven Beatmung verneint werden, oder sie lehnen diese Behandlungsmaßnahmen ab. Diese Patienten haben eine hohe Symptomlast und benötigen eine gute palliativmedizinische Versorgung [2]. Neben dieser Gruppe der schwerkranken Patienten darf aber auch die Gruppe der Patienten nicht vernachlässigt werden, die ohne ARDS und ohne invasive Beatmung auf Intensivoder Isolierstation behandelt werden. Bei diesen Patienten sollte ebenfalls auf die Symptomkontrolle geachtet werden, da bei diesen Patienten Husten (40\%), Luftnot (32\%) und Myalgien (20\%) berichtet wurden.

Sollten ähnlich wie in Italien durch $\mathrm{zu}$ hohe Patientenzahlen die vorhandenen Behandlungskapazitäten überlastet werden, sodass es zu einer Priorisierung kommen muss [3], könnte die erste Patientengruppe plötzlich sehr groß werden. 
Der Fokus in der Symptomlinderung bei COVID-19 liegt auf der Behandlung der respiratorischen Symptome [2]. Wir möchten aber gezielt die Leser von Der Schmerz aufrufen, ihre Beobachtungen zu Schmerzen bei COVID-19 zu berichten und zur Publikation bei Der Schmerz einzureichen.

Ebenso rufen wir zur Einreichung von Berichten über Beeinträchtigungen in der Behandlung von Schmerzpatienten im Rahmen der Umverteilung von Ressourcen oder von Isolierungsmaßnahmen in der Pandemie (z.B. Abbruch der Physiotherapie wegen Isolierung im Pflegeheim, Depression bei Besuchsverbot, Abbruch der multimodalen Schmerztherapie) auf.

Wir haben hierfür ein besonderes Verfahren zur Begutachtung eingerichtet, sodass wir Fallberichte, klinische Erfahrungsberichte oder epidemiologische Studien in kürzester Zeit veröffentlichen können, um einen schnellen Zugang zu diesen Erfahrungen (Online First) zu gewährleisten.

Wir rufen auch auf zur Einreichung von Fallberichten, klinischen Erfahrungsberichten, epidemiologischen oder anderen Studien zur Behandlung von anderen Symptomen außer Schmerzen, die wir in Erweiterung des Spektrums der Zeitschrift ebenfalls für ein solches schnelles Publikationsverfahren berücksichtigen werden.

Berichten Sie über Ihre Erkenntnisse, wenn sie auch für andere Behandler relevant sind, und teilen Sie Ihre Erfahrungen mit den Lesern von Der Schmerz!

\section{Korrespondenzadresse}

Prof. Dr. Lukas Radbruch

Klinik für Palliativmedizin, Universitätsklinikum Bonn

Sigmund-Freud-Str. 25, 53127 Bonn,

Deutschland

lukas.radbruch@malteser.org

Interessenkonflikt. L. Radbruch und H.-G. Schaible geben an, dass kein Interessenkonflikt besteht.

\section{Literatur}

1. Bajwah S, Wilcock A, Towers R, Costantini M, Bausewein C, Simon ST, Bendstrup E, Prentice W, Johnson MJ, Currow DC, Kreuter M, Wells AU, Birring SS, Edmonds P, Higginson IJ (2020)
Managing the supportive care needs of those affected by COVID-19. Eur Respir J. https://doi.org/ 10.1183/13993003.00815-2020

2. Deutsche Gesellschaft für Palliativmedizin (DGP), Deutsche Gesellschaft für Pneumonologie und Beatmungsmedizin (DGPneu) (2020) Therapie von Patient*innen mit COVID-19 aus palliativmedizinischerPerspektive

3. Deutsche Interdisziplinäre Vereinigung für Intensiv- und Notfallmedizin (DIVI), Deutsche Gesellschaft für Interdisziplinäre Notfall-und Akutmedizin (DGINA), Deutsche Gesellschaft für Anästhesiologie und Intensivmedizin (DGAl), Deutsche Gesellschaft für Internistische Intensivmedizin und Notfallmedizin (DGIIN), Deutsche Gesellschaft für Pneumologie und Beatmungsmedizin (DGP), Deutsche Gesellschaft für Palliativmedizin (DGP), Akademie für Ethik in der Medizin (AEM) (2020) Entscheidungen über die Zuteilung von Ressourcen in der Notfall- und der Intensivmedizin im Kontext der Covid-19-Pandemie: Klinisch ethische Empfehlungen

4. Dreher M, Kersten A, Bickenbach J, Balfanz P, Hartmann B, Cornelissen C, Daher A, Stöhr R, Kleines M, Lemmen S, Brokmann JC, Müller T, Müller-Wieland D, Marx G, Marx N (2020) Charakteristik von 50 hospitalisierten COVID-19Patienten mit und ohne ARDS. Dtsch Arztebl 117:271-278

5. Kluge $S$, Janssens U, Welte $T$, Weber-Carstens $S$, Marx G, Karagiannidis C (2020) Empfehlungen zur intensivmedizinischen Therapie von Patienten mit COVID-19. Med Klin Intensivmed Notfmed 115(3):175-177

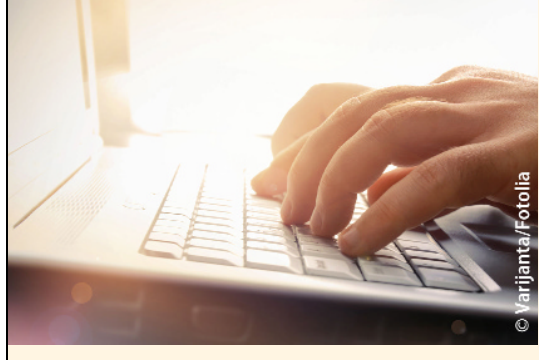

\section{Sharedlt}

Teilen Sie Beiträge von

Der Schmerz proaktiv

Möchten Sie Beiträge aus Der Schmerz unkompliziert mit Ihren Kollegen austauschen oder über Social-Media-Kanäle teilen?

Dies funktioniert dank der neuen Initiative Sharedlt von Springer Nature ganz einfach.

Jedem Abonnent von Der Schmerz steht über SpringerLink ein „Shareable link“ zur Read-Only-Version eines jeden Beitrags zur Verfügung, der unbegrenzt genutzt werden kann.

- Rufen Sie hierzu Der Schmerz unter https://link.springer.com/journal/482 auf.

- Wählen Sie den gewünschten zu teilenden Beitrag einer beliebigen Ausgabe seit 1987 an.

- In der rechten Spalte finden Sie den Menüpunkt „Share article“, hinter dem sich der "Shareable link" verbirgt.

- Teilen Sie den "Shareable Link“. Jeder der diesen „Shareable link“ nutzt, kann den entsprechenden Beitrag in einer Read-Only-Version lesen, auch Nichtabonnenten.

Nutzen Sie diese Möglichkeit, Beiträge von Der Schmerz zu teilen und in wissenschaftliche Diskussionen einzutreten. 
Hier steht eine Anzeige.

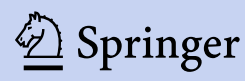

